SUPPORTING INFORMATION

\title{
Molecular Approach to the Effect of Interfacial Energy on Growth Habit of $\varepsilon$-HNIW
}

\author{
Hong-Min Shim and Kee-Kahb Koo ${ }^{*}$ \\ Department of Chemical and Biomolecular Engineering, Sogang University, Seoul 04107, Korea \\ *E-mail: koo@sogang.ac.kr
}




\section{S1. Overall Kink Rate}

The $k$ th kink rate on edge $i, u_{k}$, is defined as ${ }^{1}$

$$
\begin{gathered}
u_{k}=j^{+} P_{k}-j_{k+1}^{-} P_{k+1} \\
P_{k+2}=\left(\frac{j^{+}+j_{k+1}^{-}}{j_{k+2}^{-}}\right) P_{k+1}-\left(\frac{j^{+}}{j_{k+2}^{-}}\right) P_{k}
\end{gathered}
$$

where $j^{+}$is the flux into thermodynamic kink sites that is independent of the type of kink, $j_{\mathrm{k}+1}^{-}$ is the flux out of the $(k+1)$ st kink site, $P_{k}$ and $P_{k+1}$ are the probabilities of $k$ th and $(k+1)$ st kink sites terminated along the edge $i$, respectively, where $\sum_{k=1}^{n} P_{k}=1, n$ is the total number of types of kinks sites. To maintain the crystal stoichiometry, the overall kink rate, $u$, which is defined as number of molecules added per unit time, has the relationship ${ }^{2}$

$$
u_{1}=u_{2}=\cdots=u_{n}=u / n
$$

The step velocity is affected by not only kink density but also local concentration. ${ }^{2}$ The local concentration has a different value on different crystal face. Therefore, general equations of fluxes can be written as

$$
\begin{gathered}
j_{(h k l)}^{+}=V_{\mathrm{m}} X_{\mathrm{A}(h k l)} v_{0} \exp \left(\frac{-\Delta E}{k_{\mathrm{B}} T}\right) \\
j_{k(h k l)}^{-}=\left(1-V_{\mathrm{m}} X_{\mathrm{A}(h k l)}\right) v_{0} \exp \left(-\frac{\left(\Delta E+\Delta W_{k(h k l)}^{\mathrm{kink}}\right)}{k_{\mathrm{B}} T}\right)
\end{gathered}
$$

where $V_{\mathrm{m}}$ and $X_{\mathrm{A}(h k l)}$ are the molar volume and local concentration of solute molecules on the $h k l$ face, respectively, $v_{0}$ is a kinetic prefactor, $k_{\mathrm{B}}$ is Boltzmann constant, $T$ is temperature, $\Delta E$ is the energy barrier for transition state of a solute molecule into a kink site. In the present work, $\Delta W_{k(h k l)}^{\mathrm{kink}}$ is defined as the energy required for removing a solute molecule from the $k$ th kink site into the terrace on the $h k l$ face. The kink rate can be reduced as $u^{\prime}=u /\left[v_{0} \exp \left(-\Delta E / k_{\mathrm{B}} T\right)\right]$ leaving the only anisotropic terms because the height $\Delta E$ is regarded as a constant value regardless of crystal faces. ${ }^{3}$ In a supersaturated solution, $X_{\mathrm{A}(h k l)}$ becomes $X_{\mathrm{A}(h k l)}^{0}(\sigma+1)$ where 
$X_{\mathrm{A}(h k l)}^{0}$ is the equilibrium local concentration and $\sigma$ is the supersaturation defined by $\sigma=$ $\left(X_{\mathrm{A}} / X_{\mathrm{A}}^{0}\right)-1$.

At equilibrium $\left(j^{+}=j^{-}\right)$, the quantity $V_{\mathrm{m}} X_{\mathrm{A}(h k l)}^{0}$ is obtained using the mass balance of fluxes

$$
V_{\mathrm{m}} X_{\mathrm{A}(h k l)}^{0}=\frac{\exp \left(-\Delta W_{k(h k l)}^{\mathrm{kink}} / k_{\mathrm{B}} T\right)}{1+\exp \left(-\Delta W_{k(h k l)}^{\text {kink }} / k_{\mathrm{B}} T\right)}
$$

In the present work, the arithmetic mean value $\Delta W_{k(h k l) \text {,avg }}^{\text {kink }}$ was used.

\section{S2. Solvent Effects}

To consider the effect of solvent on the growth of crystal faces, the interfacial energy, $\gamma_{i}$, is taken into account and approximated by the regular solution model of mixing.

$$
\gamma_{i}=\gamma_{\mathrm{tot}, i}^{\mathrm{crys}}+\gamma_{\mathrm{tot}}^{\mathrm{solv}}-W_{\mathrm{ad}, i}
$$

where $\gamma_{\text {tot }, i}^{\text {cryst }}$ is the total surface energy at the kink site $i, \gamma_{\text {tot }}^{\text {solv }}$ is the surface energy of a solvent, often termed cohesion energy, and $W_{\mathrm{ad}, i}$ is the work of adhesion at the crystal-solvent interface. $W_{\mathrm{ad}, i}$ could be determined by mixing the Coulombic interactions of the crystal and the associative interactions of the solvent because polarization and hydrogen bonding interactions are inseparable in common force fields where the hydrogen bonding is practically contained in the Coulombic interactions. ${ }^{4}$

$W_{\mathrm{ad}, i}$ can be approximated by the geometric mean equation, ${ }^{5}$

$$
W_{\mathrm{ad}, i}=2 \frac{\gamma_{\mathrm{d}, i}^{\text {cryst }}}{\left|\gamma_{\mathrm{d}, i}^{\text {cryst }}\right|} \sqrt{\left|\gamma_{\mathrm{d}, i}^{\text {cryst }}\right| \gamma_{\mathrm{d}}^{\text {solv }}}+2 \frac{\gamma_{\mathrm{c}, i}^{\text {cryst }}}{\left|\gamma_{\mathrm{c}, i}^{\text {cryst }}\right|} \sqrt{\left|\gamma_{\mathrm{c}, i}^{\text {cryst }}\right| \gamma_{\mathrm{a}}^{\text {solv }}}
$$

where the subscripts $\mathrm{d}, \mathrm{c}$, and a denote the dispersive, Coulombic, and associative components of the surface energy, respectively.

The surface energy is obtained by the relation ${ }^{5,6}$ 


$$
\gamma_{\mathrm{z}}=f \delta_{\mathrm{z}}^{2} N_{A}^{-1 / 3} V_{\mathrm{m}}^{1 / 3}
$$

where $f$ is a fractional factor, $\delta_{\mathrm{z}}$ is the solubility parameter of component $\mathrm{z}$, where $\mathrm{z}=\mathrm{d}, \mathrm{p}, \mathrm{h}, \mathrm{a}$ for the dispersive, polar, hydrogen bonding, and associative components, respectively, $N_{A}$ is Avogadro's number, and $V_{\mathrm{m}}$ is the molar volume. In general, an association component of solubility parameter is described as

$$
\delta_{\mathrm{a}}=\sqrt{\delta_{\mathrm{p}}^{2}+\delta_{\mathrm{h}}^{2}}
$$

Solubility parameters for ethyl acetate and methanol are summarized in Table S1, respectively. ${ }^{7}$

Table S1. Solubility Parameters for Ethyl Acetate and Methanol

\begin{tabular}{|c|c|c|c|c|c|c|c|c|c|}
\hline solvent & $f$ & $\begin{array}{c}V_{\mathrm{m}} \\
\left(\mathrm{cm}^{3} / \mathrm{mol}\right)\end{array}$ & $\begin{array}{c}\delta_{\mathrm{d}} \\
\left(\mathrm{cal} / \mathrm{cm}^{3}\right)^{1 / 2}\end{array}$ & $\begin{array}{c}\delta_{\mathrm{p}} \\
\left(\mathrm{cal} / \mathrm{cm}^{3}\right)^{1 / 2}\end{array}$ & $\begin{array}{c}\delta_{\mathrm{h}} \\
\left(\mathrm{cal} / \mathrm{cm}^{3}\right)^{1 / 2}\end{array}$ & $\begin{array}{c}\gamma_{\mathrm{d}} \\
\left(\mathrm{erg} / \mathrm{cm}^{2}\right)\end{array}$ & $\begin{array}{c}\gamma_{\mathrm{a}} \\
\left(\mathrm{erg} / \mathrm{cm}^{2}\right)\end{array}$ & $\begin{array}{c}\gamma_{\text {tot }} \\
\left(\mathrm{erg} / \mathrm{cm}^{2}\right)\end{array}$ & $\begin{array}{c}\gamma_{\text {exp. }}{ }^{2} \\
\left(\mathrm{erg} / \mathrm{cm}^{2}\right)\end{array}$ \\
\hline ethyl acetate & 0.12 & 98.5 & 7.7 & 2.6 & 3.5 & 16.3 & 5.2 & 21.5 & 22.9 \\
\hline methanol & 0.06 & 40.7 & 7.4 & 6.0 & 10.9 & 5.6 & 15.8 & 21.4 & 22.0 \\
\hline
\end{tabular}

${ }^{a}$ Ref. 8.

The energy of formation of the $i$ th solid-fluid bond, $\phi_{i}$, is estimated by ${ }^{9,10}$

$$
\phi_{i}=\phi_{i}^{\mathrm{sf}}-\frac{1}{2}\left(\phi_{i}^{\mathrm{ss}}+\phi_{i}^{\mathrm{ff}}\right)
$$

where $\phi_{i}^{\text {ss }}$ and $\phi_{i}^{\mathrm{ff}}$ are interaction energies between solid-solid and fluid-fluid phases, respectively; $\phi_{i}^{\mathrm{ss}}=-\gamma_{i}^{\mathrm{ss}} s_{i}$ and $\phi_{i}^{\mathrm{ff}}=-\gamma_{i}^{\mathrm{ff}} s_{i}$, and the $\phi_{i}^{\mathrm{sf}}$ is a gain in surface energy by forming one solid-fluid contact; $\phi_{i}^{\mathrm{sf}}=-W_{\mathrm{ad}, i} s_{i} / 2 .^{2}$ In the present work, the area of $\varepsilon$-HNIW molecule was approximated as $s_{i}=\left(V_{\text {unit }} / Z\right)^{2 / 3}$ where $V_{\text {unit }}$ is the volume of a unit cell and $Z$ is the number of molecules in the unit cell. Interaction energies of solid-fluid are summarized in Table S2. 
Table S2. Interaction Energies of Solid-Fluid ${ }^{a}$

\begin{tabular}{|c|c|c|}
\hline bond & $\phi_{i}^{\text {EA }}(\mathrm{kcal} / \mathrm{mol})$ & $\phi_{i}^{\mathrm{Me}}(\mathrm{kcal} / \mathrm{mol})$ \\
\hline$a$ & 0.57 & 0.50 \\
\hline$b$ & 0.62 & 0.42 \\
\hline$c$ & 0.27 & 0.42 \\
\hline$d$ & 0.27 & 0.29 \\
\hline$e$ & 0.18 & 0.25 \\
\hline$f$ & 0.10 & 0.07 \\
\hline$g$ & 0.04 & 0.19 \\
\hline$h$ & 0.18 & 0.49 \\
\hline
\end{tabular}

${ }^{a}$ Superscripts EA and Me denote the solute-ethyl acetate and the solute-methanol interaction energy, respectively.

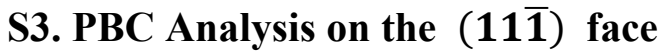

Two PBCs cannot coexist to form one edge of spirals in which a weaker PBC should be eliminated. Therefore, a four-sided spiral comprising the PBCs of $a-e$ and $b-d-c-d$ is considered in the present work. Along the [112] direction, four different rearrangements of growth units are found as shown in Figure S1. On the other hand, a manifold of rearrangements of growth units are found to exist along the [110] edge of the (111) face; see Figure S2. From the straight edge where bonds $d$ and $h$ are exposed to the [112] direction, four different rearrangements of growth units are possible; the energy expense per kink, $\phi_{\mathrm{A} 1}^{\mathrm{kink}}=-\left\{2 E_{a}+\right.$ $\left.n_{\text {edge }}\left(E_{b}+E_{c}-E_{h}\right)\right\} / 4 \quad, \quad \phi_{\mathrm{A} 2}^{\mathrm{kink}}=-\left\{E_{a}+E_{e}+n_{\mathrm{edge}}\left(E_{b}+E_{c}-E_{h}\right)\right\} / 4$, $\phi_{\mathrm{A} 3}^{\mathrm{kink}}=-\left\{2 E_{e}+n_{\text {edge }}\left(E_{b}+E_{c}-E_{h}\right)\right\} / 4$, and $\phi_{\mathrm{A} 4}^{\mathrm{kink}}=-\left\{E_{a}+E_{e}+n_{\text {edge }}\left(E_{b}+E_{c}-E_{h}\right)\right\} / 4$, respectively. The kink energy distributions for all the possible rearrangements of growth units are displayed in Figure S3. Rearrangements of growth units by breaking bonds $b$ and $c$ from the straight edge leads to creation of bonds $h$ on the edge, which is thermodynamically not preferred because the surface energy of an edge increases as growth units continues to be rearranged. However, the rearrangements by types A5-8 give constant kink energy; $\phi_{\mathrm{A} 5}^{\mathrm{kink}}=\phi_{\mathrm{A} 6}^{\mathrm{kink}}=$ 
$\phi_{\mathrm{A} 7}^{\mathrm{kink}}=\phi_{\mathrm{A} 8}^{\mathrm{kink}}=-\left(2 E_{a}+2 E_{e}+2 E_{d}\right) / 4$. The rearrangements whose kink energy gradually becomes smaller as the $n_{\text {edge }}$ increases are also found on the [1]10] edge; $\phi_{\mathrm{B} 1}^{\mathrm{kink}}=-\left\{2 E_{a}+\right.$ $\left.n_{\text {edge }}\left(E_{h}-E_{b}-E_{c}\right)\right\} / 4, \quad \phi_{\mathrm{B} 2}^{\mathrm{kink}}=-\left\{E_{a}+E_{e}+n_{\text {edge }}\left(E_{h}-E_{b}-E_{c}\right)\right\} / 4, \quad \phi_{\mathrm{B} 3}^{\mathrm{kink}}=-\left\{2 E_{e}+\right.$ $\left.n_{\text {edge }}\left(E_{h}-E_{b}-E_{c}\right)\right\} / 4$, and $\phi_{\mathrm{B} 4}^{\text {kink }}=-\left\{E_{a}+E_{e}+n_{\text {edge }}\left(E_{h}-E_{b}-E_{c}\right)\right\} / 4$, respectively. Those rearrangements tend to vigorously occur, so that an initial edge is readily transformed into another straight edge, which is not associated with formation of kink sites. In the present work, the rest of rearrangements of growth units is used for the estimation of kink density along the [11̄0] direction; $\quad \phi_{\mathrm{B} 5}^{\mathrm{kink}}=-\left(4 E_{a}+2 E_{h}\right) / 4, \quad \phi_{\mathrm{B} 6}^{\mathrm{kink}}=-\left(2 E_{a}+2 E_{e}+E_{h}\right) / 4 \quad, \quad \phi_{\mathrm{B} 7}^{\mathrm{kink}}=$ $-\left(4 E_{e}\right) / 4$, and $\phi_{\mathrm{B} 8}^{\mathrm{kink}}=-\left(2 E_{a}+2 E_{e}+E_{h}\right) / 4$, respectively because it provides a relatively higher kink density compared to that obtained by rearrangements of types $\phi_{\mathrm{A} 5}^{\mathrm{kink}}, \phi_{\mathrm{A} 6}^{\mathrm{kink}}, \phi_{\mathrm{A} 7}^{\mathrm{kink}}$, and $\phi_{\mathrm{A} 8}^{\mathrm{kink}}$.

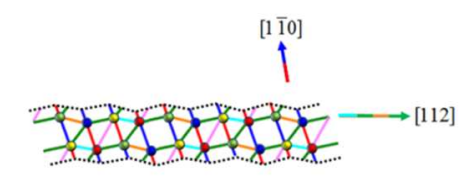

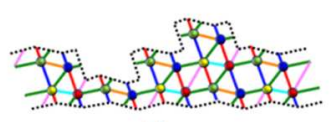

(a)

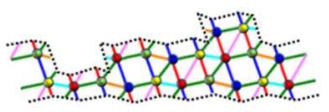

(c)

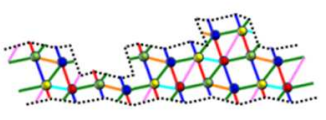

(b)

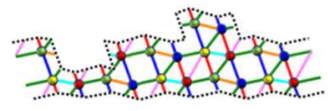

(d)

Figure S1. Rearrangements of growth units to form different types of kink sites on the [112]

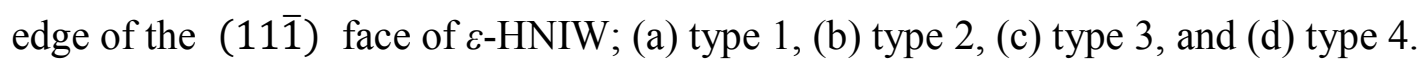




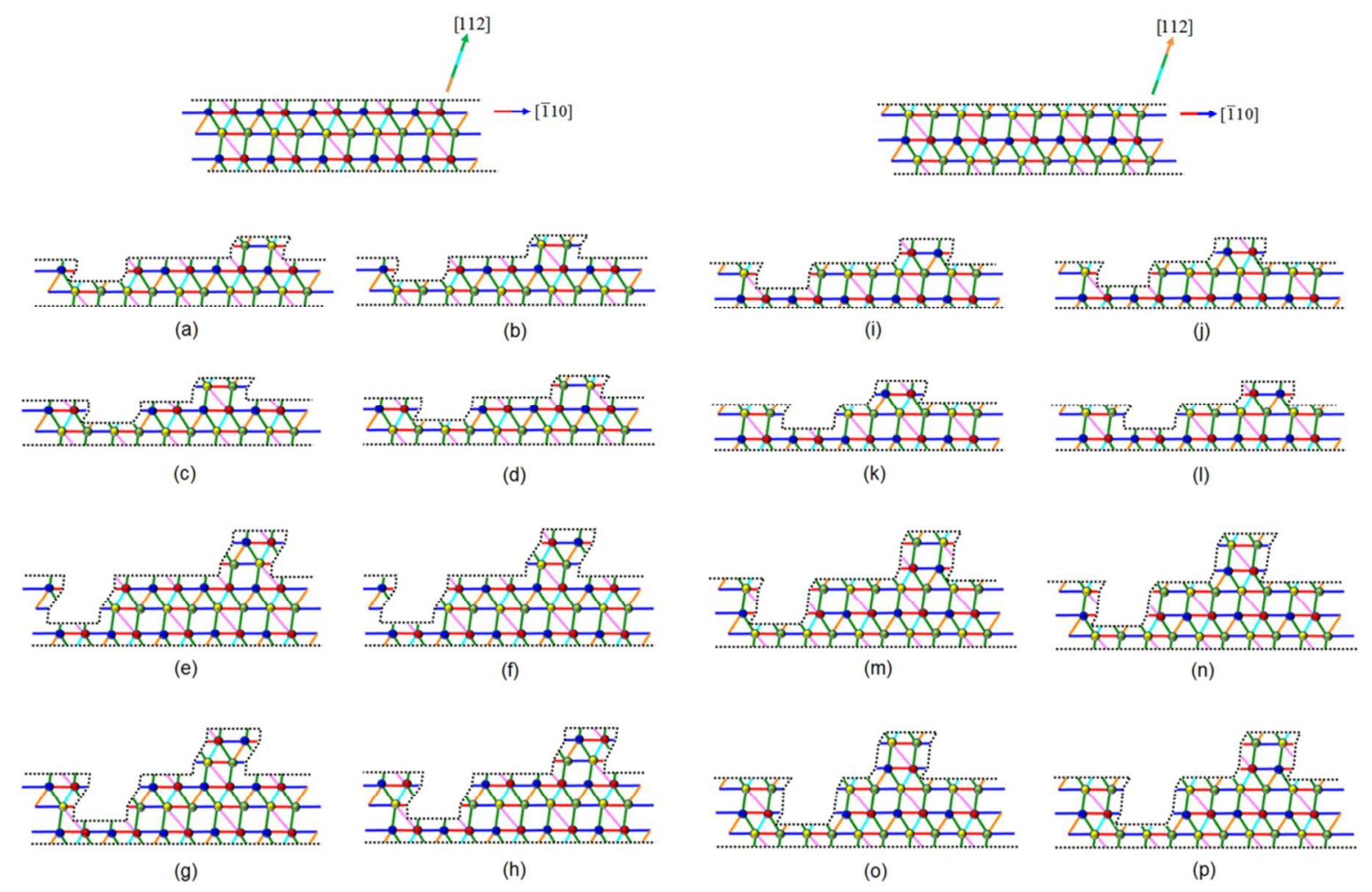

Figure S2. Rearrangements of growth units to form different types of kink sites on the [110] edge of the (111) face; (a) type A1, (b) type A2, (c) type A3, (d) type A4, (e) type A5, (f) type A6, (g) type A7, (h) type A8, (i) type B1, (j) type B2, (k) type B3, (l) type B4, (m) type B5, (n) type B6, (o) type B7, and (p) type B8, respectively. 

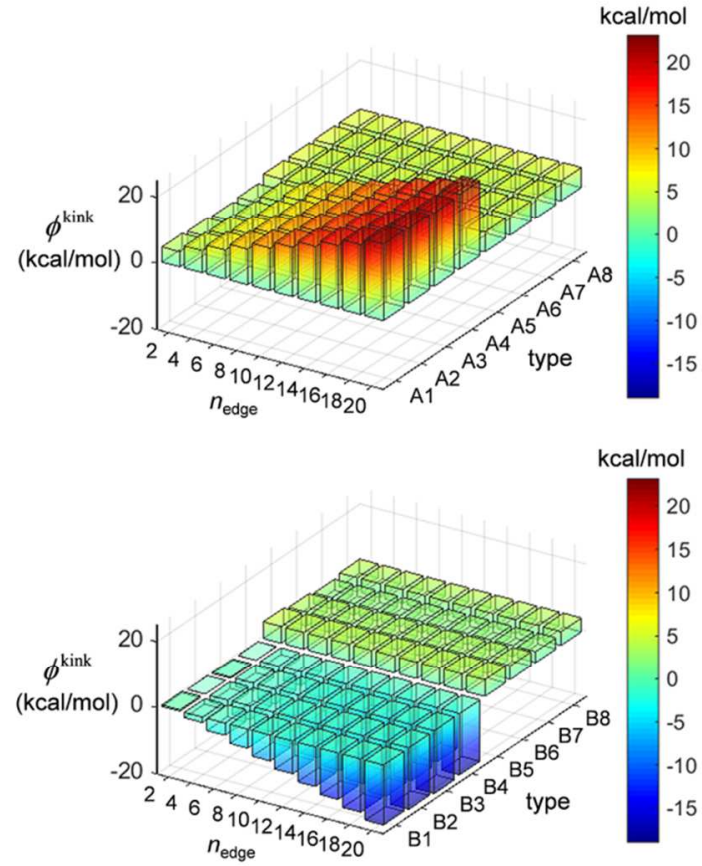

Figure S3. Distribution of kink energy on the [1110] edge of the (111) face.

\section{S4. BFDH and AE models}

Table S3. Interplanar Distance, Attachment Energy and Slice Energy of $\varepsilon$-HNIW

\begin{tabular}{|c|l|l|}
\hline face & $d_{\text {hkl }}(\AA)$ & $E_{\text {att }}(\mathrm{kcal} / \mathrm{mol})$ \\
\hline$\{011\}$ & 8.98 & -17.22 \\
\hline$\{10 \overline{1}\}$ & 8.26 & -18.21 \\
\hline$\{110\}$ & 7.03 & -18.47 \\
\hline$\{11 \overline{1}\}$ & 6.90 & -19.85 \\
\hline$\{002\}$ & 6.42 & -19.22 \\
\hline$\{020\}$ & 6.29 & -26.32 \\
\hline$\{101\}$ & 6.28 & -23.56 \\
\hline
\end{tabular}

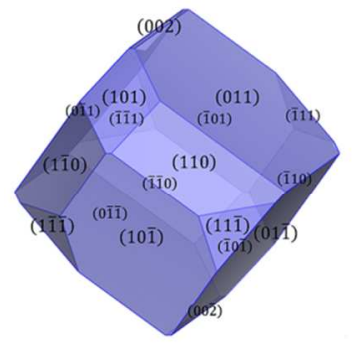

(a)

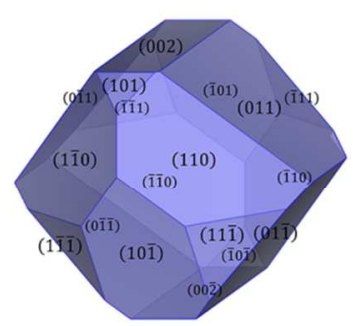

(b)

Figure S4. Predicted crystal shapes of $\varepsilon$-HNIW by the (a) BFDH and (b) AE models. 


\section{S5. Simulation Results}

All the values were calculated at $T=300 \mathrm{~K}$ and $\sigma=0.01$. The superscripts vac, EA, and Me denote the solute-vacuum, the solute-ethyl acetate, and the solute-methanol interaction energy, respectively.

Table S4. Kink Energies, Kink Densities, and Critical Length of Edges for $\varepsilon$-HNIW in Vacuum, Ethyl Acetate, and Methanol

\begin{tabular}{|c|c|c|c|c|c|c|c|c|c|c|}
\hline face & edge & $\begin{array}{c}\phi_{i}^{\text {kink,vac }} \\
(\mathrm{kcal} / \mathrm{mol})\end{array}$ & $\begin{array}{c}\phi_{i}^{\text {kink,EA }} \\
(\mathrm{kcal} / \mathrm{mol})\end{array}$ & $\begin{array}{c}\phi_{i}^{\text {kink,Me }} \\
(\mathrm{kcal} / \mathrm{mol})\end{array}$ & $\rho^{\mathrm{vac}}$ & $\rho^{\mathrm{EA}}$ & $\rho^{\mathrm{Me}}$ & $\begin{array}{l}l_{\mathrm{c}, i}^{\mathrm{vac}} \\
(\AA)\end{array}$ & $\begin{array}{l}l_{\mathrm{c}, i}^{\mathrm{EA}} \\
(\AA)\end{array}$ & $\begin{array}{l}l_{\mathrm{c}, i}^{\mathrm{Me}} \\
(\AA)\end{array}$ \\
\hline \multirow[t]{2}{*}{ (110) } & {$[\overline{1} 10]$} & 4.70 & 0.43 & 0.50 & 0.0007 & 0.4892 & 0.4606 & 12189 & 1137 & 1315 \\
\hline & [001] & $2.43 / 2.03$ & $0.38 / 0.06$ & $0.10 / 0.26$ & 0.0888 & 0.7414 & 0.7517 & 5046 & 444 & 340 \\
\hline \multirow[t]{2}{*}{$(11 \overline{1})$} & {$[\overline{1} 10]$} & $5.37 / 3.33$ & $0.69 / 0.14$ & $0.62 / 0.32$ & 0.0051 & 0.6844 & 0.6583 & 11281 & 968 & 1072 \\
\hline & {$[112]$} & $1.88 / 2.23$ & $0.12 / 0.21$ & $0.14 / 0.16$ & 0.1145 & 0.7524 & 0.7554 & 4961 & 393 & 366 \\
\hline \multirow[t]{2}{*}{ (002) } & {$[0 \overline{1} 0]$} & 2.51 & 0.27 & 0.15 & 0.0285 & 0.5596 & 0.6051 & 5335 & 573 & 336 \\
\hline & {$[100]$} & 1.15 & 0.10 & 0.06 & 0.2236 & 0.6273 & 0.6423 & 3451 & 307 & 191 \\
\hline \multirow[t]{2}{*}{$(10 \overline{1})$} & {$[\overline{1} 0 \overline{1}]$} & 1.88 & 0.11 & 0.14 & 0.0780 & 0.6221 & 0.6122 & 4373 & 268 & 326 \\
\hline & {$[010]$} & 2.51 & 0.27 & 0.15 & 0.0285 & 0.5595 & 0.6050 & 5335 & 573 & 337 \\
\hline \multirow[t]{2}{*}{ (101) } & {$[10 \overline{1}]$} & 0.52 & 0.14 & 0.33 & 0.4552 & 0.6090 & 0.5339 & 4587 & 454 & 1013 \\
\hline & {$[010]$} & 2.51 & 0.27 & 0.15 & 0.0285 & 0.5595 & 0.6050 & 5335 & 573 & 337 \\
\hline \multirow[t]{2}{*}{ (011) } & {$[\overline{1} \overline{1} 1]$} & $2.51 / 2.43$ & $0.24 / 0.36$ & $0.12 / 0.05$ & 0.0594 & 0.7074 & 0.7753 & 4594 & 555 & 161 \\
\hline & {$[100]$} & 2.65 & 0.13 & 0.21 & 0.0229 & 0.6145 & 0.5804 & 7918 & 403 & 656 \\
\hline \multirow[t]{3}{*}{ (020) } & {$[101]$} & 1.88 & 0.11 & 0.14 & 0.0780 & 0.6221 & 0.6122 & 4373 & 268 & 326 \\
\hline & {$[100]$} & 1.15 & 0.10 & 0.06 & 0.2236 & 0.6273 & 0.6423 & 3447 & 306 & 191 \\
\hline & {$[10 \overline{1}]$} & 0.52 & 0.14 & 0.33 & 0.4552 & 0.6090 & 0.5339 & 4587 & 454 & 1013 \\
\hline
\end{tabular}


Table S5. Local Concentrations and Existence Probabilities for $\varepsilon$-HNIW in Vacuum, Ethyl Acetate, and Methanol

\begin{tabular}{|c|c|c|c|c|c|c|c|c|c|c|c|}
\hline face & edge & $\begin{array}{c}\text { site, } \\
k\end{array}$ & $\begin{array}{c}\Delta W_{k(h k l)}^{\mathrm{kink}, \mathrm{vac}} \\
(\mathrm{kcal} / \mathrm{mol})\end{array}$ & $P_{k}^{\text {vac }}$ & $\begin{array}{c}\Delta W_{k(h k l)}^{\text {kink,EA }} \\
(\mathrm{kcal} / \mathrm{mol})\end{array}$ & $P_{k}^{\mathrm{EA}}$ & $\begin{array}{c}\Delta W_{k(h k l)}^{\mathrm{kink}, \mathrm{Me}} \\
(\mathrm{kcal} / \mathrm{mol})\end{array}$ & $P_{k}^{\mathrm{Me}}$ & $V_{\mathrm{m}} X_{\mathrm{A}(h k l)}^{0, \mathrm{vac}}$ & $V_{\mathrm{m}} X_{\mathrm{A}(h k l)}^{0, \mathrm{EA}}$ & $V_{\mathrm{m}} X_{\mathrm{A}(h k l)}^{0, \mathrm{Me}}$ \\
\hline \multirow[t]{6}{*}{ (110) } & \multirow[t]{4}{*}[\overline{1}10]{} & 1 & 5.98 & 0.24 & 1.07 & 0.26 & 0.97 & 0.20 & \multirow[t]{6}{*}{0.000095} & \multirow[t]{6}{*}{0.160040} & \multirow[t]{6}{*}{0.146483} \\
\hline & & 2 & 5.06 & 0.11 & 0.89 & 0.22 & 1.12 & 0.23 & & & \\
\hline & & 3 & 6.43 & 0.52 & 1.12 & 0.28 & 1.22 & 0.31 & & & \\
\hline & & 4 & 4.61 & 0.11 & 0.84 & 0.22 & 0.87 & 0.24 & & & \\
\hline & \multirow[t]{2}{*}{ [001] } & 1 & 3.69 & 0.04 & 0.45 & 0.29 & 0.68 & 0.35 & & & \\
\hline & & 2 & 7.35 & 0.96 & 1.51 & 0.71 & 1.41 & 0.65 & & & \\
\hline \multirow[t]{8}{*}{$(11 \overline{1})$} & \multirow[t]{4}{*}[\overline{1}10]{} & 1 & 5.98 & 0.38 & 1.07 & 0.32 & 0.97 & 0.25 & \multirow[t]{8}{*}{0.000169} & \multirow[t]{8}{*}{0.174912} & \multirow[t]{8}{*}{0.155297} \\
\hline & & 2 & 3.55 & 0.02 & 0.45 & 0.14 & 0.55 & 0.11 & & & \\
\hline & & 3 & 6.77 & 0.39 & 1.31 & 0.28 & 1.71 & 0.37 & & & \\
\hline & & 4 & 4.44 & 0.19 & 0.85 & 0.25 & 0.79 & 0.26 & & & \\
\hline & \multirow[t]{4}{*}[112]{} & 1 & 5.58 & 0.44 & 0.73 & 0.25 & 0.97 & 0.32 & & & \\
\hline & & 2 & 3.55 & 0.02 & 0.45 & 0.11 & 0.55 & 0.15 & & & \\
\hline & & 3 & 6.83 & 0.41 & 1.47 & 0.28 & 1.22 & 0.20 & & & \\
\hline & & 4 & 4.74 & 0.11 & 1.03 & 0.34 & 1.28 & 0.33 & & & \\
\hline \multirow[t]{3}{*}{$(002)$} & \multirow[t]{2}{*}[0\overline{1}0]{} & 1 & 3.67 & 0.06 & 0.68 & 0.43 & 0.57 & 0.40 & \multirow[t]{3}{*}{0.000130} & \multirow[t]{3}{*}{0.190513} & \multirow[t]{3}{*}{0.199198} \\
\hline & & 2 & 7.00 & 0.94 & 1.04 & 0.57 & 1.08 & 0.60 & & & \\
\hline & {$[100]$} & 1 & 5.33 & 1.00 & 0.86 & 1.00 & 0.82 & 1.00 & & & \\
\hline \multirow[t]{4}{*}{$(10 \overline{1})$} & \multirow[t]{2}{*}[\overline{1}0\overline{1}]{} & 1 & 6.77 & 0.88 & 1.31 & 0.60 & 1.71 & 0.68 & \multirow[t]{4}{*}{0.000085} & \multirow[t]{4}{*}{0.140102} & \multirow[t]{4}{*}{0.108277} \\
\hline & & 2 & 4.40 & 0.12 & 0.85 & 0.40 & 0.79 & 0.32 & & & \\
\hline & \multirow[t]{2}{*}[010]{} & 1 & 6.43 & 0.80 & 1.12 & 0.52 & 1.22 & 0.49 & & & \\
\hline & & 2 & 4.74 & 0.20 & 1.03 & 0.48 & 1.28 & 0.51 & & & \\
\hline \multirow[t]{4}{*}{$(101)$} & \multirow[t]{2}{*}[10\overline{1}]{} & 1 & 3.03 & 0.12 & 0.61 & 0.37 & 0.69 & 0.41 & \multirow[t]{4}{*}{0.000799} & \multirow[t]{4}{*}{0.173332} & 0.178505 \\
\hline & & 2 & 5.46 & 0.88 & 1.24 & 0.63 & 1.12 & 0.59 & & & \\
\hline & {$[010]$} & 1 & 3.03 & 0.12 & 0.61 & 0.37 & 0.69 & 0.41 & & & \\
\hline & & 2 & 5.46 & 0.88 & 1.24 & 0.63 & 1.12 & 0.59 & & & \\
\hline (011) & {$[\overline{1} \overline{1} 1]$} & 1 & 3.92 & 0.24 & 0.90 & 0.51 & 0.99 & 0.53 & 0.000423 & 0.184496 & 0.178392 \\
\hline & & 2 & 5.33 & 0.76 & 0.86 & 0.49 & 0.82 & 0.46 & & & \\
\hline & {$[100]$} & 1 & 5.33 & 0.76 & 0.86 & 0.49 & 0.82 & 0.46 & & & \\
\hline & & 2 & 3.92 & 0.24 & 0.90 & 0.51 & 0.99 & 0.53 & & & \\
\hline$(020)$ & {$[101]$} & 1 & 3.56 & 1.00 & 0.42 & 1.00 & 0.56 & 1.00 & 0.002540 & 0.329198 & 0.278566 \\
\hline & {$[100]$} & 1 & 3.56 & 1.00 & 0.42 & 1.00 & 0.56 & 1.00 & & & \\
\hline & {$[10 \overline{1}]$} & 1 & 3.56 & 1.00 & 0.42 & 1.00 & 0.56 & 1.00 & & & \\
\hline
\end{tabular}


Table S6. Interfacial Edge Energies, Effective Edge Energies, and Onset Supersaturations for $\varepsilon$-HNIW in Vacuum, Ethyl Acetate, and Methanol

\begin{tabular}{|c|c|c|c|c|c|c|c|c|c|c|}
\hline face & edge & $\begin{array}{c}\gamma_{i}^{\text {edge,vac }} \\
\left(\mathrm{erg} / \mathrm{cm}^{2}\right)\end{array}$ & $\begin{array}{l}\gamma_{i}^{\text {edge,EA }} \\
\left(\mathrm{erg} / \mathrm{cm}^{2}\right)\end{array}$ & $\begin{array}{l}\gamma_{i}^{\text {edge,Me }} \\
\left(\mathrm{erg} / \mathrm{cm}^{2}\right)\end{array}$ & $\begin{array}{c}\phi_{\mathrm{e}}^{\mathrm{vac}} \\
(\mathrm{kcal} / \mathrm{mol})\end{array}$ & $\begin{array}{c}\phi_{\mathrm{e}}^{\mathrm{EA}} \\
(\mathrm{kcal} / \mathrm{mol})\end{array}$ & $\begin{array}{c}\phi_{\mathrm{e}}^{\mathrm{Me}} \\
(\mathrm{kcal} / \mathrm{mol})\end{array}$ & $\sigma_{2 \mathrm{D}}^{\mathrm{vac}}$ & $\sigma_{2 \mathrm{D}}^{\mathrm{EA}}$ & $\sigma_{2 \mathrm{D}}^{\mathrm{Me}}$ \\
\hline \multirow[t]{2}{*}{ (110) } & {$[\overline{1} 10]$} & $70.6 / 105.7$ & $16.3 / 35.2$ & $17.7 / 35.7$ & \multirow[t]{2}{*}{6.72} & \multirow[t]{2}{*}{2.02} & \multirow[t]{2}{*}{2.07} & \multirow[t]{2}{*}{28.25} & \multirow[t]{2}{*}{1.64} & \multirow[t]{2}{*}{1.74} \\
\hline & {$[001]$} & 96.9 & 29.8 & 30.7 & & & & & & \\
\hline \multirow[t]{2}{*}{$(11 \overline{1})$} & {$[\overline{1} 10]$} & $53.7 / 107.6$ & $8.7 / 36.3$ & $10.5 / 36.0$ & \multirow[t]{2}{*}{6.20} & \multirow[t]{2}{*}{1.75} & \multirow[t]{2}{*}{1.80} & \multirow[t]{2}{*}{23.14} & \multirow[t]{2}{*}{1.19} & \multirow[t]{2}{*}{1.27} \\
\hline & [112] & 87.7 & 25.0 & 25.9 & & & & & & \\
\hline \multirow[t]{2}{*}{$(002)$} & {$[0 \overline{1} 0]$} & 96.9 & 29.7 & 30.8 & \multirow[t]{2}{*}{6.73} & \multirow[t]{2}{*}{2.11} & \multirow[t]{2}{*}{2.15} & \multirow[t]{2}{*}{29.77} & \multirow[t]{2}{*}{1.93} & \multirow[t]{2}{*}{1.99} \\
\hline & {$[100]$} & 102.0 & 33.2 & 32.9 & & & & & & \\
\hline \multirow[t]{2}{*}{$(10 \overline{1})$} & {$[\overline{1} 0 \overline{1}]$} & 90.4 & 26.3 & 27.1 & \multirow[t]{2}{*}{6.71} & \multirow[t]{2}{*}{1.82} & \multirow[t]{2}{*}{1.99} & \multirow[t]{2}{*}{28.43} & \multirow[t]{2}{*}{1.33} & \multirow[t]{2}{*}{1.61} \\
\hline & {$[010]$} & 82.0 & 21.0. & 24.1 & & & & & & \\
\hline \multirow[t]{2}{*}{ (101) } & {$[10 \overline{1}]$} & 91.1 & 29.1 & 25.6 & \multirow[t]{2}{*}{4.73} & \multirow[t]{2}{*}{1.13} & \multirow[t]{2}{*}{1.03} & \multirow[t]{2}{*}{14.28} & \multirow[t]{2}{*}{0.63} & \multirow[t]{2}{*}{0.50} \\
\hline & {$[010]$} & 60.9 & 13.0 & 12.0 & & & & & & \\
\hline \multirow[t]{2}{*}{ (011) } & {$[\overline{1} \overline{1} 1]$} & 60.5 & 11.5 & 13.2 & 4.90 & 0.94 & 1.05 & 13.36 & 0.29 & 0.38 \\
\hline & [100] & $26.0 / 72.9$ & $0.9 / 18.0$ & $2.9 / 17.8$ & & & & & & \\
\hline$(020)$ & {$[101]$} & 53.7 & 8.7 & 10.4 & 4.50 & 0.89 & 1.00 & 7.79 & 0.19 & 0.25 \\
\hline & [100] & 59.9 & 11.1 & 13.3 & & & & & & \\
\hline & {$[10 \overline{1}]$} & 74.1 & 18.5 & 18.4 & & & & & & \\
\hline
\end{tabular}


Table S7. Anisotropic Structure Factors, Step Velocities, and Relative Growth Rates for $\varepsilon$ HNIW in Vacuum, Ethyl Acetate, and Methanol ${ }^{a}$

\begin{tabular}{|c|c|c|c|c|c|c|c|c|c|c|c|}
\hline face & edge & $\begin{array}{l}a_{\mathrm{e}} \\
(\AA)\end{array}$ & $\begin{array}{l}a_{\mathrm{p}} \\
(\AA)\end{array}$ & $\begin{array}{c}\text { angle } \\
\text { (radian) }\end{array}$ & $v^{\prime \text { vac }}$ & $v^{\prime \mathrm{EA}}$ & $v^{\prime \mathrm{Me}}$ & $\begin{array}{l}d_{h k l} \\
(\AA)\end{array}$ & $R^{\text {vac }}$ & $R^{\mathrm{EA}}$ & $R^{\mathrm{Me}}$ \\
\hline \multirow[t]{4}{*}{ (110) } & {$[\overline{1} 10]$} & 7.6 & 13.2 & 1.74 & $6.41 \cdot 10^{-9}$ & $1.22 \cdot 10^{-2}$ & $1.02 \cdot 10^{-2}$ & \multirow[t]{4}{*}{7.03} & \multirow[t]{4}{*}{1.0} & \multirow[t]{4}{*}{1.0} & \multirow[t]{4}{*}{1.0} \\
\hline & [001] & 6.7 & 7.5 & 1.40 & $1.09 \cdot 10^{-7}$ & $8.87 \cdot 10^{-3}$ & $8.94 \cdot 10^{-3}$ & & & & \\
\hline & [1]̄0] & 7.6 & 13.2 & 1.74 & $6.41 \cdot 10^{-9}$ & $1.22 \cdot 10^{-2}$ & $1.02 \cdot 10^{-2}$ & & & & \\
\hline & {$[00 \overline{1}]$} & 6.7 & 7.5 & 1.40 & $1.09 \cdot 10^{-7}$ & $8.87 \cdot 10^{-3}$ & $8.94 \cdot 10^{-3}$ & & & & \\
\hline \multirow[t]{4}{*}{$(11 \overline{1})$} & {$[\overline{1} 10]$} & 7.6 & 13.4 & 1.91 & $3.93 \cdot 10^{-8}$ & $1.78 \cdot 10^{-2}$ & $1.35 \cdot 10^{-2}$ & \multirow[t]{4}{*}{6.90} & \multirow[t]{4}{*}{6.0} & \multirow[t]{4}{*}{1.38} & \multirow[t]{4}{*}{1.26} \\
\hline & {$[112]$} & 7.1 & 7.2 & 1.23 & $4.45 \cdot 10^{-7}$ & $9.64 \cdot 10^{-3}$ & $8.96 \cdot 10^{-3}$ & & & & \\
\hline & {$[1 \overline{1} 0]$} & 7.6 & 13.4 & 1.91 & $3.93 \cdot 10^{-8}$ & $1.78 \cdot 10^{-2}$ & $1.35 \cdot 10^{-2}$ & & & & \\
\hline & {$[\overline{1} \overline{1} \overline{2}]$} & 7.1 & 7.2 & 1.23 & $4.45 \cdot 10^{-7}$ & $9.64 \cdot 10^{-3}$ & $8.96 \cdot 10^{-3}$ & & & & \\
\hline \multirow[t]{4}{*}{ (002) } & {$[0 \overline{1} 0]$} & 6.2 & 8.8 & 1.57 & $7.16 \cdot 10^{-8}$ & $1.14 \cdot 10^{-2}$ & $1.27 \cdot 10^{-2}$ & \multirow[t]{4}{*}{6.42} & \multirow[t]{4}{*}{15.8} & \multirow[t]{4}{*}{1.16} & \multirow[t]{4}{*}{3.34} \\
\hline & {$[100]$} & 8.8 & 6.2 & 1.57 & $1.82 \cdot 10^{-6}$ & $9.28 \cdot 10^{-3}$ & $1.00 \cdot 10^{-2}$ & & & & \\
\hline & {$[010]$} & 6.2 & 8.8 & 1.57 & $7.16 \cdot 10^{-8}$ & $1.14 \cdot 10^{-2}$ & $1.27 \cdot 10^{-2}$ & & & & \\
\hline & {$[\overline{100]}$} & 8.8 & 6.2 & 1.57 & $1.82 \cdot 10^{-6}$ & $9.28 \cdot 10^{-3}$ & $1.00 \cdot 10^{-2}$ & & & & \\
\hline \multirow[t]{4}{*}{$(10 \overline{1})$} & {$[\overline{1} 0 \overline{1}]$} & 6.8 & 6.2 & 1.57 & $1.78 \cdot 10^{-7}$ & $6.15 \cdot 10^{-3}$ & $4.05 \cdot 10^{-3}$ & \multirow[t]{4}{*}{8.26} & \multirow[t]{4}{*}{14.5} & \multirow[t]{4}{*}{1.40} & \multirow[t]{4}{*}{1.41} \\
\hline & [010] & 6.2 & 6.8 & 1.57 & $1.05 \cdot 10^{-7}$ & $6.26 \cdot 10^{-3}$ & $5.05 \cdot 10^{-3}$ & & & & \\
\hline & [101] & 6.8 & 6.2 & 1.57 & $1.78 \cdot 10^{-7}$ & $6.15 \cdot 10^{-3}$ & $4.05 \cdot 10^{-3}$ & & & & \\
\hline & {$[0 \overline{1} 0]$} & 6.2 & 6.8 & 1.57 & $1.05 \cdot 10^{-7}$ & $6.26 \cdot 10^{-3}$ & $5.05 \cdot 10^{-3}$ & & & & \\
\hline \multirow[t]{4}{*}{ (101) } & {$[10 \overline{1}]$} & 9.0 & 6.2 & 1.57 & $9.37 \cdot 10^{-6}$ & $7.51 \cdot 10^{-3}$ & $7.07 \cdot 10^{-3}$ & \multirow[t]{4}{*}{6.28} & \multirow[t]{4}{*}{$\sim 10^{2}$} & \multirow[t]{4}{*}{1.18} & \multirow[t]{4}{*}{1.17} \\
\hline & [010] & 6.2 & 9.0 & 1.57 & $8.46 \cdot 10^{-7}$ & $9.92 \cdot 10^{-3}$ & $1.15 \cdot 10^{-2}$ & & & & \\
\hline & {$[\overline{101]}$} & 9.0 & 6.2 & 1.57 & $9.37 \cdot 10^{-6}$ & $7.51 \cdot 10^{-3}$ & $7.07 \cdot 10^{-3}$ & & & & \\
\hline & 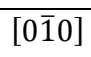 & 6.2 & 9.0 & 1.57 & $8.46 \cdot 10^{-7}$ & $9.92 \cdot 10^{-3}$ & $1.15 \cdot 10^{-2}$ & & & & \\
\hline \multirow[t]{4}{*}{ (011) } & {$[\overline{1} \overline{1} 1]$} & 5.5 & 7.2 & 0.95 & $1.31 \cdot 10^{-6}$ & $1.16 \cdot 10^{-2}$ & $1.21 \cdot 10^{-2}$ & \multirow[t]{4}{*}{8.98} & \multirow[t]{4}{*}{$\sim 10^{2}$} & 3.19 & 4.06 \\
\hline & [100] & 8.8 & 4.4 & 2.19 & $6.26 \cdot 10^{-7}$ & $1.25 \cdot 10^{-2}$ & $1.13 \cdot 10^{-2}$ & & & & \\
\hline & {$[11 \overline{1}]$} & 5.5 & 7.2 & 0.95 & $1.31 \cdot 10^{-6}$ & $1.16 \cdot 10^{-2}$ & $1.21 \cdot 10^{-2}$ & & & & \\
\hline & {$[\overline{1} 00]$} & 8.8 & 4.4 & 2.19 & $6.26 \cdot 10^{-7}$ & $1.25 \cdot 10^{-2}$ & $1.13 \cdot 10^{-2}$ & & & & \\
\hline$(020)$ & [101] & 6.8 & 8.2 & 1.94 & $1.64 \cdot 10^{-5}$ & $2.52 \cdot 10^{-2}$ & $1.95 \cdot 10^{-2}$ & 6.29 & $\sim 10^{3}$ & 3.56 & 2.05 \\
\hline & {$[100]$} & 8.8 & 6.4 & 2.34 & $3.66 \cdot 10^{-5}$ & $1.97 \cdot 10^{-2}$ & $1.59 \cdot 10^{-2}$ & & & & \\
\hline & {$[10 \overline{1}]$} & 9.0 & 6.2 & 2.00 & $7.28 \cdot 10^{-5}$ & $1.87 \cdot 10^{-2}$ & $1.29 \cdot 10^{-2}$ & & & & \\
\hline & {$[\overline{1} 0 \overline{1}]$} & 6.8 & 8.2 & 1.94 & $1.64 \cdot 10^{-5}$ & $2.52 \cdot 10^{-2}$ & $1.95 \cdot 10^{-2}$ & & & & \\
\hline & {$[\overline{1} 00]$} & 8.8 & 6.4 & 2.34 & $3.66 \cdot 10^{-5}$ & $1.97 \cdot 10^{-2}$ & $1.59 \cdot 10^{-2}$ & & & & \\
\hline & {$[\overline{101]}$} & 9.0 & 6.2 & 2.00 & $7.28 \cdot 10^{-5}$ & $1.87 \cdot 10^{-2}$ & $1.29 \cdot 10^{-2}$ & & & & \\
\hline
\end{tabular}

${ }^{a}$ Step velocity was obtained by the relation $v_{i}^{\prime}=a_{\mathrm{p}, i} u_{i}^{\prime} \rho_{i}$. Relative growth rates were calculated by the spiral growth model. 


\section{S6. References}

(1) Kuvadia, Z. B.; Doherty, M. F. Cryst. Growth Des. 2011, 11, 2780-2802.

(2) Shim, H.-M.; Myerson, A. S.; Koo, K.-K. Cryst. Growth Des. 2016, 16, 3454-3464.

(3) Shim, H.-M.; Koo, K.-K. Cryst. Growth Des. 2014, 14, 1802-1810.

(4) Lovette, M. A.; Doherty, M. F. Cryst. Growth Des. 2012, 12, 656-669.

(5) Lovette, M. A.; Doherty, M. F. Cryst. Growth Des. 2013, 13, 3341-3352.

(6) Winn, D.; Doherty, M. F. AIChE J. 1998, 44, 2501-2514.

(7) Barton, A. F. M. Chem. Rev. 1975, 75, 731-753.

(8) Koenhen, D. M.; Smolders, C. A. J. Appl. Polym. Sci. 1975, 19, 1163-1179.

(9) Bennema, P J. Cryst. Growth 1996, 166, 17-28.

(10) Winn, D.; Doherty, M. F. AIChE J. 2000, 46, 1348-1367. 